\title{
Chapter 6 \\ Future Directions and Topics for Further Research into the Globalization of Science Curricula
}

\begin{abstract}
The impacts of globalization on science education and curricula are of considerable interest internationally, not least in terms of preparing a nation's students for employment in a rapidly changing world. This study was not a measure of the total science curriculum for each country considered, but a measure of the similarity of their intended science curriculum to the Trends in Mathematics and Science Study (TIMSS) framework; further research into the effects on the science curricula of countries that have not participated in TIMSS or using data from other relevant large-scale assessments would add an additional dimension to understanding the globalization of science curricula. Research exploring the processes by which countries embark on science curriculum reform would be an important avenue for further work in order to gain a better understanding of why countries decide to make the changes they do.
\end{abstract}

Keyword Globalization - Science curriculum • Science education Trends in Mathematics and Science Study (TIMSS)

Making use of the extensive IEA TIMSS datasets has enabled us to empirically address a number of important questions pertaining to the globalization of science curricula over a wide time range. The research complements and builds on previous research on globalization of curricula, which has tended to focus on mathematics rather than science.

However, globalization of science curricula is a broad topic, and we were unable to explore all areas of interest given our limited resources and time. As a consequence, we here identify important areas for additional research to build further on this work.

Firstly, we focused primarily on countries' intended science curricula, as opposed to the implemented or achieved curricula. Devising a complementary research program to explore the extent to which countries' implemented and achieved curricula may be converging would be the next logical step, and the TIMSS teacher questionnaire is an obvious source of relevant data. 
Secondly, our focus was on the TIMSS science topics included in countries' curricula, as opposed to the skills emphasized within curricula. There would be value in investigating how these aspects of curricula change in countries over time; for example, whether there is a trend for an increasing emphasis on science investigation and problem-solving skills, as opposed to pure content knowledge.

Thirdly, we compared countries' science curricula using the TIMSS science framework. Whilst this framework covers a broad range of science topics, it is not an exhaustive list. It is therefore possible that some countries' science curricula included topics that were not covered in the TIMSS science framework. One way to overcome this limitation would be to conduct a study that makes direct comparisons of countries' science curricula using the original curriculum documents (as opposed to the TIMSS science framework) as the basis for the comparison.

Our research identified a number of changes in countries' science curricula over time, particularly in terms of the science content included. However, we did not explore the causal mechanisms or drivers supporting the development of curriculum changes at an international level. Research exploring the processes by which countries embark on science curriculum reform would be an important avenue for further work, in order to gain a better understanding of why countries decide to make the changes they do.

We investigated globalization in science curricula using the 1999 TIMSS survey as a baseline for Grade 8 and the 2003 TIMSS survey as a baseline for Grade 4. As discussed in Chap. 2, Rutkowski and Rutkowski (2009) have suggested that investigating globalization in education and the curriculum using data from the 1990s onwards is starting too late, because the effects of globalization were apparent far earlier than this. Extending the study of globalization back further in time may detect changes and convergence in science curricula that our study may have missed, but depends upon the availability of reliable comparative data.

Here, we focused on countries that participated in TIMSS. It could be argued that countries participating in such studies have, to some extent, already bought into a global education agenda, and thus research that explores and compares the curricula of countries participating in TIMSS against the curricula of those that do not would provide an alternative perspective and add an additional dimension to the study of the globalization of science curricula. Furthermore, as many developing countries do not participate in TIMSS, analysis that makes use of available curriculum data from these countries would augment any study of the globalization of science curricula.

Finally, our research has not made reference to, or used data from, other international large-scale assessments of science such as the Programme for International Student Assessment (PISA). Given its influence and prominence, and the addition of new PISA assessments (such as the PISA-based test for schools and PISA for Development), research which considers globalization in science curricula within the context of PISA and its assessment frameworks, would also provide important complementary information. 


\section{Summary of the Key Findings}

- The literature review identified a number of factors contributing to globalization in science education and science curricula. These included the growing emphasis on education as a mechanism for economic growth, the increase in information technology in education and the role of international large-scale assessments in facilitating comparisons between countries. Despite these factors, however, there were other forces which mediated the globalization of science curricula, such as local culture and local interpretation of a centralized curriculum.

- The analysis found strong evidence that there have been changes in intended science curricula over the last 20 years. For example, every country analyzed in the investigation had made changes, although the extent of the changes varied considerably. The analyses also identified that more changes were made to intended science curricula at Grade 4 than at Grade 8 , therefore suggesting a more fluid science curriculum in the primary phase.

- The analyses suggest that, in some respects, science curricula are becoming increasingly similar over time. For example, there was a trend for more of the TIMSS science topics to be included in the majority (over $80 \%$ ) of participating countries' curricula. This trend was observed at both grades, although was more pronounced at Grade 4.

- The cluster and discriminant analyses indicate strong convergence in the science curricula of countries participating at Grade 8. The number of countries clustering into Group 2 grew at the expense of the number clustering into Group 1. This suggests that, over time, changes in intended science curricula mean an increasing number of countries with similar profiles in terms of the topics they do and do not include in their science curricula.

- At Grade 4, the strength of evidence for science curricula becoming increasingly similar over time is less strong, with the results of the discriminant analysis suggesting that changes in countries' science curricula have led to only mild convergence in curricula.

- At both Grade 4 and Grade 8 two distinct groups of countries could be identified based on their intended science curricula. At Grade 4 one group tended to have a broad and balanced science curriculum covering a range of topics, whilst the other group had less emphasis on physical science topics and a greater focus on life sciences topics.

- Similarly, at Grade 8, one group included a greater number of TIMSS science topics in its intended curriculum than the other. However, the topics omitted from the group which included a more restricted number of science topics in their curriculum were not confined to one specific area of science, unlike Grade 4. 
- Finally the analysis identified core science topics that were present in the intended curricula of the vast majority of countries included in this analysis. At Grade 4, these core topics tended to be concentrated in the life sciences. By contrast, at Grade 8 , they were distributed more evenly across the science subjects.

\section{Reference}

Rutkowski, L., \& Rutkowski, D. (2009). Trends in TIMSS responses over time: Evidence of global forces in education? Educational Research and Evaluation, 15(2), 137-152.

Open Access This chapter is licensed under the terms of the Creative Commons Attribution-NonCommercial 4.0 International License (http://creativecommons.org/licenses/by-nc/ $4.0 /$ ), which permits any noncommercial use, sharing, adaptation, distribution and reproduction in any medium or format, as long as you give appropriate credit to the original author(s) and the source, provide a link to the Creative Commons license and indicate if changes were made.

The images or other third party material in this chapter are included in the chapter's Creative Commons license, unless indicated otherwise in a credit line to the material. If material is not included in the chapter's Creative Commons license and your intended use is not permitted by statutory regulation or exceeds the permitted use, you will need to obtain permission directly from the copyright holder.

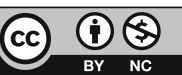

